\title{
Calibration methods for microwave free space measurements
}

\author{
I. Rolfes and B. Schiek
}

Institut für Hochfrequenztechnik, Ruhr-Universität Bochum, Universitätsstraße 150, 44801 Bochum, Germany

\begin{abstract}
In this article calibration methods for the precise, contact-less measurement of the permittivity, permeability or humidity of materials are presented. The free space measurement system principally consists of a pair of focusing horn-lens antennas connected to the ports of a vector network analyzer. Based on the measured scattering parameters, the dielectric material parameters are calculable. Due to systematic errors as e.g. transmission losses of the cables or mismatches of the antennas, a calibration of the measurement setup is necessary. For this purpose calibration methods with calibration standards of equal mechanical lengths are presented. They have the advantage, that the measurement setup can be kept in a fixed position, for example no displacement of the antennas is needed. The presented self-calibration methods have in common that the calibration structures consist of a so-called obstacle network which can be partly unknown. The obstacle can either be realized as a transmissive or a reflective network depending on the chosen method. An increase of the frequency bandwidth is achievable with the reflective realization. The theory of the calibration methods and some experimental results will be presented.
\end{abstract}

\section{Introduction}

At microwave frequencies the permittivity, permeability or humidity of materials can be determined from measurements of the scattering parameters. For materials realized as planar probes the parameters can be measured contact-less in free space (Ghodgaonkar et al., 1989). The free space measurement system which is depicted in Fig. 1 basically consists of a vector network analyzer (VNA) connected to a pair of spot-focusing horn-lens-antennas.

The use of lenses aims at bundling the radiated electromagnetic waves between the antennas where the material under test will be placed. As the measurement results are af-

Correspondence to: I. Rolfes

(ilona.rolfes@rub.de)

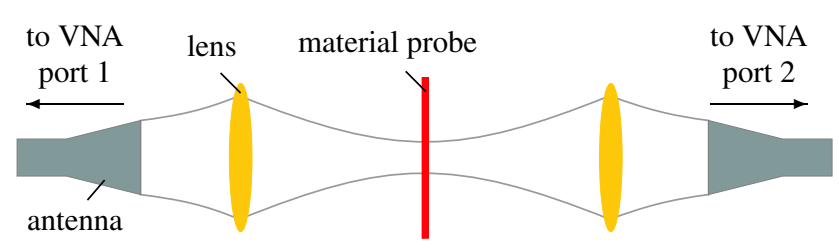

Fig. 1. Setup of the free space measurement system.

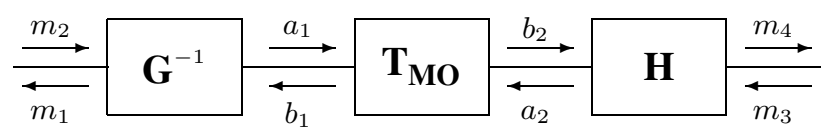

Fig. 2. Error model of the measurement system with a four-channel vector network analyzer.

fected by different systematic errors, which are caused e.g. by transmission losses of the cables or the mismatches of the antennas, it is necessary to calibrate the setup. For this purpose the whole measurement system can be described with the help of an error model known from the calibration of vector network analyzers with four measurement channels, as shown in Fig. 2.

The error transmission matrices $\mathbf{G}$ and $\mathbf{H}$ which represent the systematic errors have to be calculated during the calibration. It is advantageous to use self-calibration procedures where some parameters of the calibration circuits can be partly unknown. For the measurements in free space, the well-known TRL-method (Through Reflect Line) (Engen and Hoer, 1979) has the drawback that its calibration standards are of different mechanical lengths. For the realization of the line standard for instance the antennas have to be displaced, as can be seen in Fig. 3 .

Such a variation of the antenna positions might be critical due to changes of the beam propagation. It is thus more advantageous to use self-calibration techniques where the standards are all of equal mechanical lengths as will be presented in the following. The described methods are 

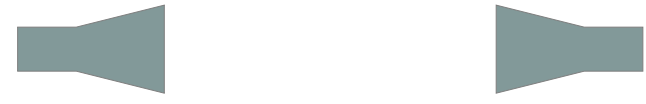

$\mathrm{T}$
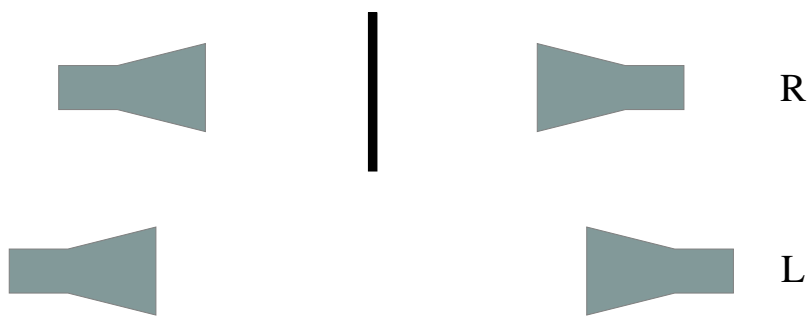

$\mathrm{L}$

Fig. 3. TRL-calibration of the free space system.

principally based on calibration standards which consist of a so-called obstacle network. It will be distinguished between methods based on transmissive calibration standards: LNN (Heuermann and Schiek, 1997), $\mathrm{L}_{1} \mathrm{~L}_{2} \mathrm{NN}$ and $\mathrm{LN}_{1} \mathrm{~N}_{2}$ method (Rolfes and Schiek, 2002), and methods with reflective standards which might have a weak transmission: LRR (Rolfes and Schiek, 2003), $\mathrm{L}_{1} \mathrm{~L}_{2} \mathrm{RR}$ and $\mathrm{LR}_{1} \mathrm{R}_{2}$ method. In addition, the theory of the reflective methods can be adopted to the well-known TRM-method (Eul and Schiek, 1991) resulting in a fairly brief derivation.

\section{Transmissive calibration methods}

The calibration structures of the LNN-, $\mathrm{L}_{1} \mathrm{~L}_{2} \mathrm{NN}$ and the $\mathrm{LN}_{1} \mathrm{~N}_{2}$-method are all based on transmissive networks.

\subsection{LNN method}

The LNN-structure consists of an obstacle network which has to be placed at three consecutive positions in equal distances as shown in Fig. 4. The obstacle can e.g. be realized as a dielectric plate.

The calibration structures are described with the help of transmission matrices with $\mathbf{L}$ representing the line element of length $l$ with the unknown propagation constant $\gamma$ and $\mathbf{Q}$ standing for the obstacle network.

$\mathbf{L}=\left[\begin{array}{cc}k & 0 \\ 0 & k^{-1}\end{array}\right], \quad \mathbf{Q}=\left[\begin{array}{ll}q_{11} & q_{12} \\ q_{21} & q_{22}\end{array}\right]$

The obstacles have to be symmetrical $\left(q_{12}=-q_{21}\right)$ and reciprocal $\left(q_{11} q_{22}-q_{12} q_{21}=1\right)$ and are assumed without loss of generality to be of the electrical length zero. The calibration circuits are described by matrices $\mathbf{M}_{\mathbf{i}}$ with $i=0,1,2,3$ which are known from measurements.

$$
\mathbf{M}_{\mathbf{0}}=\mathbf{G}^{-1} \mathbf{L L H}, \quad \mathbf{M}_{\mathbf{1}}=\mathbf{G}^{-1} \mathbf{L L Q H}
$$$$
\mathbf{M}_{2}=\mathbf{G}^{-1} \mathbf{L Q L H}, \quad \mathbf{M}_{\mathbf{3}}=\mathbf{G}^{-1} \mathbf{Q L L H}
$$

$\mathbf{G}$ and $\mathbf{H}$ are two-ports which represent the systematic errors of the VNA. During the self-calibration $\mathbf{G}$ and $\mathbf{H}$ are eliminated in order to determine the unknown line- and obstacle-
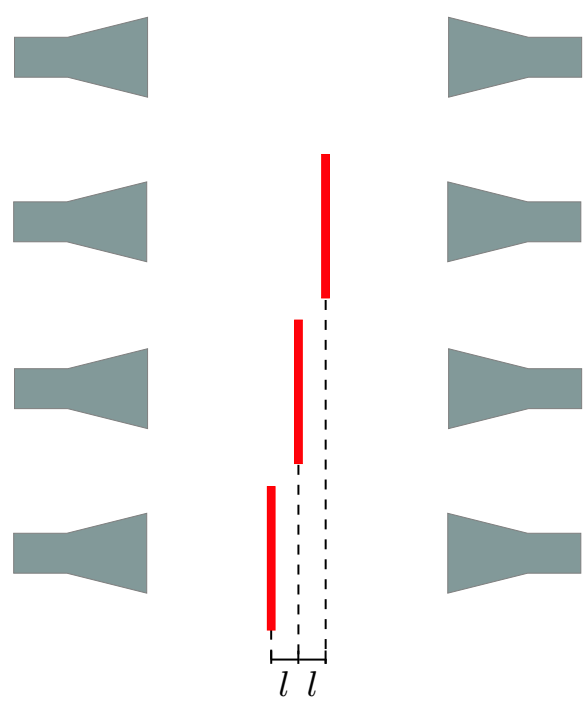

Fig. 4. Setup of the LNN calibration structures.

parameters. The following equation results i.e. for the matrices $\mathbf{M}_{\mathbf{0}}$ and $\mathbf{M}_{\mathbf{1}}$, using the similarity transformation of the trace function abbreviated as $t r$ :

$$
\begin{aligned}
\alpha_{1} & =\operatorname{tr}\left\{\mathbf{M}_{\mathbf{1}} \mathbf{M}_{\mathbf{0}}^{-1}\right\}=\operatorname{tr}\left\{\mathbf{G}^{-1} \mathbf{L L Q H}\left(\mathbf{G}^{-1} \mathbf{L L H}\right)^{-1}\right\} \\
& =\operatorname{tr}\{\mathbf{Q}\}=q_{11}+q_{22}
\end{aligned}
$$

With $\alpha_{2}=\operatorname{tr}\left\{\mathbf{M}_{2} \mathbf{M}_{1}^{-1}\right\}, \alpha_{3}=\operatorname{tr}\left\{\mathbf{M}_{3} \mathbf{M}_{1}^{-1}\right\}$ and $\alpha=$ $\left(\alpha_{3}-2\right)\left(\alpha_{2}-2\right)^{-1}$ the unknown parameters are calculable as follows

$k= \pm \frac{\sqrt{\alpha}}{2} \pm \sqrt{\frac{\alpha}{4}-1}, \quad q_{12}=-q_{21}= \pm \sqrt{\frac{\alpha_{2}-2}{\left(k-\frac{1}{k}\right)^{2}}}$

$q_{11}=\frac{\alpha_{1}}{2} \pm \sqrt{\frac{\alpha_{1}^{2}}{4}+q_{12}^{2}-1}, \quad q_{22}=\alpha_{1}-q_{11}$

An approximate knowledge of the structures dimensions is necessary in order to choose the correct solutions.

\subsection{Extended LNN method}

An extension of the $\mathrm{LNN}$ method is the $\mathrm{L}_{1} \mathrm{~L}_{2} \mathrm{NN}$ method with either equal or non-equal unknown distances $l_{1}$ and $l_{2}$ between the obstacle positions. The advantage of this variant is that the positioning of the obstacles becomes quite uncritical, because the obstacles do not have to be placed in precisely equal distances from each other. The theoretical derivation of this method is very similar to the one of the LNN method. Instead of one line matrix $\mathbf{L}$ two line matrices $\mathbf{L}_{\mathbf{1}}$ and $\mathbf{L}_{\mathbf{2}}$ have to be considered in Eqs. (2) and (3), so that with the trace functions

$\beta_{1}=\operatorname{tr}\left\{\mathbf{M}_{\mathbf{1}} \mathbf{M}_{\mathbf{0}}^{-1}\right\}, \beta_{2}=\operatorname{tr}\left\{\mathbf{M}_{\mathbf{3}} \mathbf{M}_{\mathbf{2}}^{-1}\right\}, \beta_{3}=$ $\operatorname{tr}\left\{\mathbf{M}_{2} \mathbf{M}_{1}^{-1}\right\}, \beta_{4}=\operatorname{tr}\left\{\mathbf{M}_{3} \mathbf{M}_{1}^{-1}\right\}$ the line and obstacle parameters can be determined as follows:

$k_{2}^{2}+k_{2}\left(\frac{\beta_{6}}{\beta_{5}^{2}}-\beta_{6}-\frac{1}{\beta_{6}}\right)+1=0, k_{1}=\frac{-\beta_{6}}{\beta_{5}\left(\beta_{6} k_{2}-1\right)}$ 

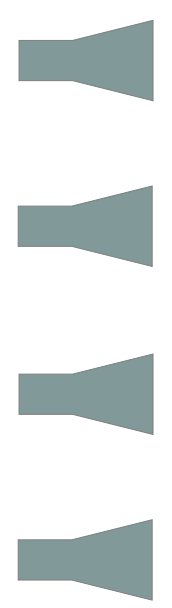
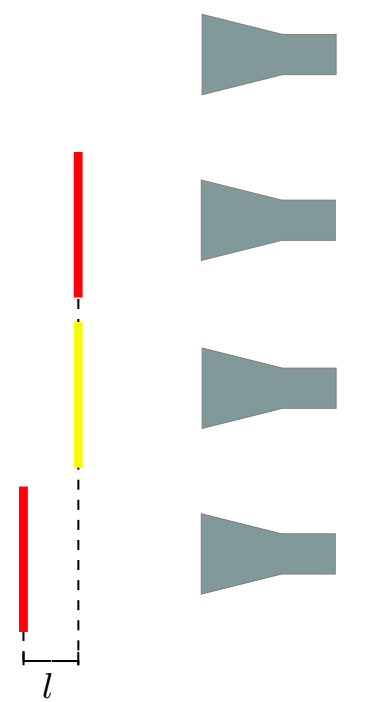

Fig. 5. Setup of the $\mathrm{LN}_{1} \mathrm{~N}_{2}$ calibration structures.

$q_{12}=-q_{21}= \pm \sqrt{\frac{\beta_{2}-2}{\left(k_{1}-k_{1}^{-1}\right)^{2}}}$

$q_{11}=\frac{\beta_{1}}{2} \pm \sqrt{\frac{\beta_{1}^{2}}{4}+q_{12}^{2}-1}, \quad q_{22}=\beta_{1}-q_{11}$

with

$\beta_{5}^{2}=\frac{\beta_{2}-2}{\beta_{3}-2}=\frac{\left(k_{1}-k_{1}^{-1}\right)^{2}}{\left(k_{2}-k_{2}^{-1}\right)^{2}}$

$\beta_{6}^{2}=\frac{\beta_{2}-2}{\beta_{4}-2}=\frac{\left(k_{1}-k_{1}^{-1}\right)^{2}}{\left(k_{1} k_{2}-k_{1}^{-1} k_{2}^{-1}\right)^{2}}$

As already pointed out for the LNN method an approximate knowledge of the calibration circuits dimension is necessary.

\section{$2.3 \quad \mathrm{LN}_{1} \mathrm{~N}_{2}$ method}

Furthermore the calibration on the basis of two different transmissive obstacle networks is realizable with the $\mathrm{LN}_{1} \mathrm{~N}_{2}$ method. The calibration structures for a free space system are shown in Fig. 5.

The two different obstacle networks can be described with the transmission matrices $\mathbf{A}$ and $\mathbf{B}$.

$\mathbf{A}=\left[\begin{array}{ll}a_{11} & a_{12} \\ a_{21} & a_{22}\end{array}\right], \quad \mathbf{B}=\left[\begin{array}{ll}b_{11} & b_{12} \\ b_{21} & b_{22}\end{array}\right]$

For the determination of the unknown parameters the trace functions of the measurement matrices of Fig. 5 can be written as follows:

$$
\begin{aligned}
\gamma_{a} & =\operatorname{tr}\left\{\mathbf{M}_{\mathbf{1}} \mathbf{M}_{\mathbf{0}}^{-1}\right\}=\operatorname{tr}\{\mathbf{A}\} \\
\gamma_{b}=\operatorname{tr}\left\{\mathbf{M}_{\mathbf{2}} \mathbf{M}_{\mathbf{0}}^{-1}\right\} & =\operatorname{tr}\{\mathbf{B}\} \\
\gamma_{a b}=\operatorname{tr}\left\{\mathbf{M}_{\mathbf{2}} \mathbf{M}_{\mathbf{1}}^{-1}\right\} & =\operatorname{tr}\left\{\mathbf{A} \mathbf{B}^{-1}\right\} \\
\gamma_{a l} & =\operatorname{tr}\left\{\mathbf{M}_{\mathbf{3}} \mathbf{M}_{\mathbf{1}}^{-1}\right\}=\operatorname{tr}\left\{\mathbf{A} \mathbf{L} \mathbf{A}^{-1} \mathbf{L}^{-1}\right\} \\
\gamma_{a b l}=\operatorname{tr}\left\{\mathbf{M}_{\mathbf{3}} \mathbf{M}_{\mathbf{2}}^{-1}\right\} & =\operatorname{tr}\left\{\mathbf{A} \mathbf{L} \mathbf{B}^{-1} \mathbf{L}^{-1}\right\}
\end{aligned}
$$

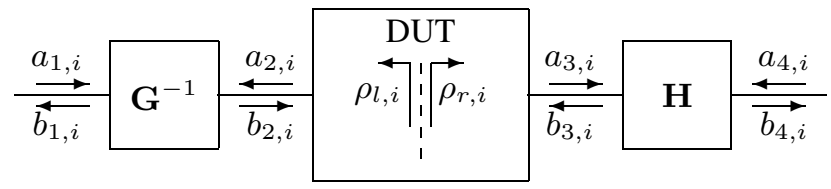

Fig. 6. Simplified block diagram of the analyzer setup.

On the basis of this system of equations the obstacle and line parameters are calculable similar to the previous solutions (Rolfes and Schiek, 2002). The calibration standards of the $\mathrm{LN}_{1} \mathrm{~N}_{2}$ method have the advantage that only two obstacle positions are needed instead of three for the LNN method. The $\mathrm{LN}_{1} \mathrm{~N}_{2}$ calibration standards require thus less space. In addition the obstacles can be placed symmetrically around the focusing area of the antenna-lens setup.

\section{Reflective methods}

While the previously presented methods have in common that the obstacles must be transmissive, the following procedures are based on obstacles either without transmission or with only a weak transmission. The topologies of the LRR, $L_{1} L_{2} R R$ and $L_{1} R_{2}$ calibration structures are principally identical to the previous ones. The obstacles can e.g. be realized as a metal plate. However, in order to reduce multiple reflections it is convenient to reduce somewhat the reflection coefficient of the obstacle by coating the metal plate with absorbing material. Due to the lack of transmission the calibration structures cannot be described on the basis of transmission matrices. Figure 6 shows a simplified block diagram of the analyzer setup with the two error two-ports $\mathbf{G}$ and $\mathbf{H}$ and the reflection coefficients $\rho_{l, i}$ and $\rho_{r, i}$ referring to the $i$ different reflective networks.

For the different methods it can be distinguished between two cases. In the first one, the obstacles are assumed to show no transmission at all and in the second one, the obstacles might or might not show a weak transmission. Depending on the realized calibration structures the appropriate way should be chosen in order to improve the accuracy.

The theories of the LRR and the $\mathrm{L}_{1} \mathrm{~L}_{2} \mathrm{RR}$ methods have already been presented in (Rolfes and Schiek, 2003). The application of the LRR method in a free space system will be discussed in some more detail in Sect. 5. In the following the $\mathrm{LR}_{1} \mathrm{R}_{2}$ method with transmission-free obstacles is presented.

\section{1 $\quad \mathrm{LR}_{1} \mathrm{R}_{2}$ method without transmission}

For this variant the free space calibration structures consist of two different reflective obstacle networks which are reflection symmetrical and have to be placed at two positions as shown in Fig. 5. Although the calibration procedure already works on the basis of four calibration structures, it is more convenient for the algebraic derivation to consider one further calibration structure where the obstacle $\mathbf{B}$ is placed on the left position. 
The calibration structures are described with the help of the line parameter $k=e^{-\gamma l}$ and the reflection coefficients $\rho_{a}$ and $\rho_{b}$. Based on the setup in Figs. 5 and 6 the reflection coefficients $\rho_{l i}$ and $\rho_{r i}$ are defined as follows:

$\rho_{l, 1}=k^{2} \rho_{a}, \rho_{l, 2}=k^{2} \rho_{b}, \rho_{l, 3}=\rho_{a}, \rho_{l, 4}=\rho_{b}$

$\rho_{r, 1}=\rho_{a}, \rho_{r, 2}=\rho_{b}, \rho_{r, 3}=k^{2} \rho_{a}, \rho_{r, 4}=k^{2} \rho_{b}$

The first calibration structure, the through connection, can be written in dependence of the transmission matrix $\mathbf{L}$ with $\mathbf{M}_{\mathbf{0}}=\mathbf{G}^{-1} \mathbf{L H}$. During the self-calibration it is further on the aim to eliminate the error two-ports $\mathbf{G}$ and $\mathbf{H}$ in order to determine the unknown parameters $k, \rho_{a}$ and $\rho_{b}$. For this purpose the error two-port $\mathbf{G}^{-1}$ is described by the following equation with $\tilde{\mathbf{G}}=\mathbf{G}^{-1}$ :

$\left[\begin{array}{c}b_{1, i} \\ a_{1, i}\end{array}\right]=\tilde{\mathbf{G}}\left[\begin{array}{l}a_{2, i} \\ b_{2, i}\end{array}\right]=\tilde{\mathbf{G}}\left[\begin{array}{c}\rho_{l, i} b_{2, i} \\ b_{2, i}\end{array}\right]$

resulting in a bilinear transformation, also known as Möbiustransformation, for the measurement value $v_{l, i}$ :

$v_{l, i}=\frac{b_{1, i}}{a_{1, i}}=\frac{\tilde{G}_{11} \rho_{l, i} b_{2, i}+\tilde{G}_{12} b_{2, i}}{\tilde{G}_{21} \rho_{l, i} b_{2, i}+\tilde{G}_{22} b_{2, i}}=\frac{\tilde{G}_{11} \rho_{l, i}+\tilde{G}_{12}}{\tilde{G}_{21} \rho_{l, i}+\tilde{G}_{22}}$

Such a bilinear transformation is generally defined as,

$x_{j}=\frac{C_{1} y_{j}+C_{2}}{C_{3} y_{j}+C_{4}}$

where the two variables $x_{j}$ and $y_{j}$ correspond to the measurement value $v_{l, i}$ and the unknown calibration standard parameter $\rho_{l, i}$ and the constants $C_{1}, \ldots, C_{4}$ represent the error two-port parameters. Concerning the two-port $\mathbf{H}$ a similar equation can be found:

$\left[\begin{array}{l}a_{4, i} \\ b_{4, i}\end{array}\right]=\mathbf{H}^{-1}\left[\begin{array}{l}b_{3, i} \\ a_{3, i}\end{array}\right]=\mathbf{H}^{-1}\left[\begin{array}{c}b_{3, i} \\ \rho_{r, i} b_{3, i}\end{array}\right]$

With $\mathbf{H}^{-1}=\mathbf{M}_{\mathbf{0}}{ }^{-1} \mathbf{G}^{-1} \mathbf{L}$ Eq. (23) can be rewritten in dependence of $\tilde{\mathbf{G}}$ :

$\mathbf{M}_{\mathbf{0}}\left[\begin{array}{l}a_{4, i} \\ b_{4, i}\end{array}\right]=\left[\begin{array}{c}a_{4, i}^{\prime} \\ b_{4, i}^{\prime}\end{array}\right]=\tilde{\mathbf{G}} \mathbf{L}\left[\begin{array}{c}b_{3, i} \\ \rho_{r, i} b_{3, i}\end{array}\right]=\tilde{\mathbf{G}}\left[\begin{array}{c}k b_{3, i} \\ \rho_{r, i} k^{-1} b_{3, i}\end{array}\right]$

In this way, another bilinear transformation in the error two-port parameter $\tilde{\mathbf{G}}$ results with $\tilde{\rho}_{r, i}=k^{2} \rho_{r, i}^{-1}$.

$$
\begin{aligned}
v_{r, i} & =\frac{a_{4, i}^{\prime}}{b_{4, i}^{\prime}}=\frac{\tilde{G}_{11} k b_{3, i}+\tilde{G}_{12} \rho_{r, i} k^{-1} b_{3, i}}{\tilde{G}_{21} k b_{3, i}+\tilde{G}_{22} \rho_{r, i} k^{-1} b_{3, i}} \\
& =\frac{\tilde{G}_{11} k^{2} \rho_{r, i}^{-1}+\tilde{G}_{12}}{\tilde{G}_{21} k^{2} \rho_{r, i}^{-1}+\tilde{G}_{22}}=\frac{\tilde{G}_{11} \tilde{\rho}_{r, i}+\tilde{G}_{12}}{\tilde{G}_{21} \tilde{\rho}_{r, i}+\tilde{G}_{22}}
\end{aligned}
$$

On the basis of the measurement of four reflection coefficients, four equations of the type of Eqs. (21) and (25) result, so that the unknown error two-port parameters $\tilde{G}_{11}, \tilde{G}_{12}, \tilde{G}_{21}$ and $\tilde{G}_{22}$ can be eliminated. This can be performed with the help of the cross ratio

$$
\frac{\left(y_{1}-y_{2}\right)\left(y_{3}-y_{4}\right)}{\left(y_{1}-y_{4}\right)\left(y_{3}-y_{2}\right)}=\frac{\left(x_{1}-x_{2}\right)\left(x_{3}-x_{4}\right)}{\left(x_{1}-x_{4}\right)\left(x_{3}-x_{2}\right)}
$$

which generally holds for a bilinear transformation as given in Eq. (22). A set of equations can thus be constructed, which only depends on the unknown reflection coefficients $\rho_{a}$ and $\rho_{b}$ and the unknown line parameter $k$ in dependence of the measurement values $v_{j}$ as e.g.:

$$
\begin{aligned}
v_{1} & =\frac{\left(v_{r, 3}-v_{r, 4}\right)\left(v_{l, 3}-v_{l, 4}\right)}{\left(v_{r, 3}-v_{l, 4}\right)\left(v_{l, 3}-v_{r, 4}\right)} \\
& =\frac{\left(\tilde{\rho}_{r, 3}-\tilde{\rho}_{r, 4}\right)\left(\rho_{l, 3}-\rho_{l, 4}\right)}{\left(\tilde{\rho}_{r, 3}-\rho_{l, 4}\right)\left(\rho_{l, 3}-\tilde{\rho}_{r, 4}\right)}=\frac{\left(\rho_{a}-\rho_{b}\right)^{2}}{\left(1-\rho_{a} \rho_{b}\right)^{2}} \\
v_{2} & =\frac{\left(v_{l, 3}-v_{r, 3}\right)\left(v_{r, 1}-v_{l, 1}\right)}{\left(v_{l, 3}-v_{l, 1}\right)\left(v_{r, 1}-v_{r, 3}\right)}=\frac{k^{2}}{\rho_{a}^{2}} \cdot \frac{\left(1-\rho_{a}^{2}\right)^{2}}{\left(1-k^{2}\right)^{2}} \\
v_{3} & =\frac{\left(v_{l, 4}-v_{r, 4}\right)\left(v_{r, 2}-v_{l, 2}\right)}{\left(v_{l, 4}-v_{l, 2}\right)\left(v_{r, 2}-v_{r, 4}\right)}=\frac{k^{2}}{\rho_{b}^{2}} \cdot \frac{\left(1-\rho_{b}^{2}\right)^{2}}{\left(1-k^{2}\right)^{2}} \\
v_{4} & =\frac{\left(v_{l, 3}-v_{r, 3}\right)\left(v_{l, 2}-v_{l, 4}\right)}{\left(v_{l, 3}-v_{l, 4}\right)\left(v_{l, 2}-v_{r, 3}\right)}=\frac{\rho_{b}\left(\rho_{a}^{2}-1\right)\left(k^{2}-1\right)}{\left(\rho_{a}-\rho_{b}\right)\left(k^{2} \rho_{a} \rho_{b}-1\right)}
\end{aligned}
$$

After some algebraic manipulation the following equations for the determination of the line parameter and the reflection coefficients result:

$$
\begin{aligned}
\rho_{a} & =-\frac{w_{3}}{2} \pm \sqrt{\frac{w_{3}^{2}}{4}-1}, \quad \rho_{b}=\frac{\rho_{a}-w_{1}}{1-w_{1} \rho_{a}} \\
k^{2} & =\frac{v_{4}\left(\rho_{a}-\rho_{b}\right)-\rho_{b}\left(\rho_{a}^{2}-1\right)}{v_{4}\left(\rho_{a}-\rho_{b}\right) \rho_{a} \rho_{b}-\rho_{b}\left(\rho_{a}^{2}-1\right)}
\end{aligned}
$$

with

$w_{1}^{2}=v_{1}, \quad w_{2}^{2}=\frac{v_{2}}{v_{3}}, \quad w_{3}=\frac{w_{2}\left(1-w_{1}^{2}\right)-\left(1+w_{1}^{2}\right)}{w_{1}}$

\subsection{The $\mathrm{LR}_{1} \mathrm{R}_{2}$ method with a weak transmission}

This algorithm is based on the description of the obstacle networks with pseudo-transmission matrices. According to Fig. 6 the measurement matrix can be defined as follows,

$\mathbf{M}_{\mathbf{i}}=\left[\begin{array}{ll}b_{1, i}^{\prime} & b_{1, i}^{\prime \prime} \\ a_{1, i}^{\prime} & a_{1, i}^{\prime \prime}\end{array}\right] \frac{1}{a_{4, i}^{\prime} b_{4, i}^{\prime \prime}-a_{4, i}^{\prime \prime} b_{4, i}^{\prime}}\left[\begin{array}{cc}b_{4, i}^{\prime \prime} & -a_{4, i}^{\prime \prime} \\ -b_{4, i}^{\prime} & a_{4, i}^{\prime}\end{array}\right]$

where the primes indicate from which side of the setup the generator signal is fed in. The determinant $\Delta m=a_{4, i}^{\prime} b_{4, i}^{\prime \prime}-$ $a_{4, i}^{\prime \prime} b_{4, i}^{\prime}$ might become zero without any transmission. The reflective structures can thus not be described on the basis of transmission matrices. They have to be represented by pseudo-transmission matrices. These pseudo-transmission matrices are constructed by multiplying the measurement matrices with the determinants $\Delta m_{a j}, \Delta m_{b j}, j=1,2$. The resulting finite part of the matrix is named $\tilde{\mathbf{M}}_{\mathbf{i}}$.

$$
\begin{array}{lll}
\mathbf{M}_{\mathbf{1}}=\frac{1}{\Delta m_{a 1}} \tilde{\mathbf{M}}_{\mathbf{1}} & \Rightarrow & \tilde{\mathbf{M}}_{\mathbf{1}}=\mathbf{G}^{-1} \mathbf{L} \Delta m_{a 1} \mathbf{A H} \\
\mathbf{M}_{\mathbf{2}}=\frac{1}{\Delta m_{b 1}} \tilde{\mathbf{M}}_{\mathbf{2}} & \Rightarrow & \tilde{\mathbf{M}}_{\mathbf{2}}=\mathbf{G}^{-1} \mathbf{L} \Delta m_{b 1} \mathbf{B H} \\
\mathbf{M}_{\mathbf{3}}=\frac{1}{\Delta m_{a 2}} \tilde{\mathbf{M}}_{\mathbf{3}} & \Rightarrow & \tilde{\mathbf{M}}_{\mathbf{3}}=\mathbf{G}^{-1} \Delta m_{a 2} \mathbf{A L H} \\
\mathbf{M}_{\mathbf{4}}=\frac{1}{\Delta m_{b 2}} \tilde{\mathbf{M}}_{\mathbf{4}} & \Rightarrow & \tilde{\mathbf{M}}_{\mathbf{4}}=\mathbf{G}^{-1} \Delta m_{b 2} \mathbf{B} \mathbf{L H}
\end{array}
$$




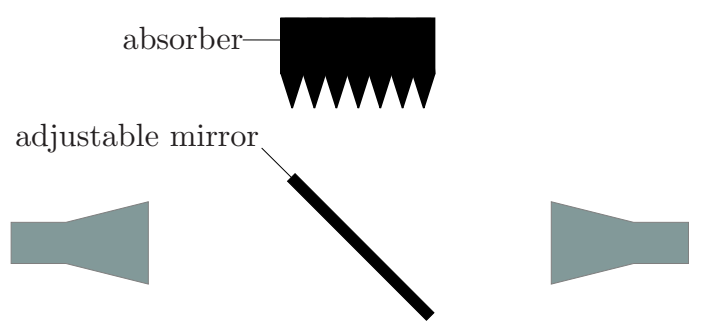

Fig. 7. Realization of the Match for the right antenna.

The product of the determinant and the obstacle transmission matrix is called pseudo-transmission matrix. With the general relation between a transmission matrix $\mathbf{T}$ and the scattering parameters $S_{11}, S_{12}, S_{21}, S_{22}$

$\mathbf{T}=\frac{1}{S_{21}}\left[\begin{array}{cc}S_{12} S_{21}-S_{11}^{2} & S_{11} \\ -S_{11} & 1\end{array}\right]$

the pseudo-transmission matrices can be written as follows:

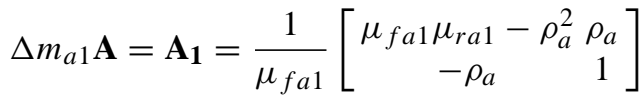

$$
\begin{aligned}
& \Delta m_{b 1} \mathbf{B}=\mathbf{B}_{\mathbf{1}}=\frac{1}{\mu_{f b 1}}\left[\begin{array}{cc}
\mu_{f b 1} \mu_{r b 1}-\rho_{b}^{2} & \rho_{b} \\
-\rho_{b} & 1
\end{array}\right] \\
& \Delta m_{a 2} \mathbf{A}=\mathbf{A}_{2}=\frac{1}{\mu_{f a 2}}\left[\begin{array}{cc}
\mu_{f a 2} \mu_{r a 2} & -\rho_{a}^{2} \\
-\rho_{a} & 1
\end{array}\right]
\end{aligned}
$$

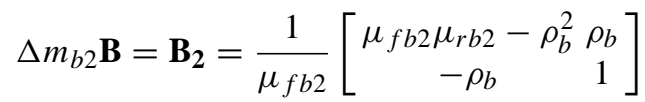

The calibration structures can thus be described on the basis of 11 parameters: $\mu_{f a 1}, \mu_{r a 1}, \mu_{f b 1}, \mu_{r b 1}, \mu_{f a 2}, \mu_{r a 2}$, $\mu_{f b 2}, \mu_{r b 2}, \rho_{a}, \rho_{b}$ and $k$ under the condition of symmetry: $S_{a, 11}=\rho_{a}=S_{a, 22}, S_{b, 11}=\rho_{b}=S_{b, 22}$. With the reciprocity condition it follows:

$S_{a, 21}=\Delta m_{a i} \mu_{f a i}=S_{a, 12}=\frac{\mu_{r a i}}{\Delta m_{a i}}, i=1,2$
$S_{b, 21}=\Delta m_{b i} \mu_{f b i}=S_{b, 12}=\frac{\mu_{r b i}}{\Delta m_{b i}}, i=1,2$

and thus

$\frac{\mu_{r a i}}{\mu_{f a i}}=\Delta m_{a i}^{2}, \quad \frac{\mu_{r b i}}{\mu_{f b i}}=\Delta m_{b i}^{2}, i=1,2$

$\mu_{f a 2}=\frac{\Delta m_{a 1}}{\Delta m_{a 2}} \mu_{f a 1}, \quad \mu_{f b 2}=\frac{\Delta m_{b 1}}{\Delta m_{b 2}} \mu_{f b 1}$.

In order to determine the unknown parameters the following trace equations are constructed eliminating $\mathbf{G}$ and $\mathbf{H}$ :

$$
\begin{aligned}
& \beta_{1}=\operatorname{tr}\left\{\tilde{\mathbf{M}}_{\mathbf{1}} \mathbf{M}_{\mathbf{0}}{ }^{-1}\right\}=\operatorname{tr}\left\{\mathbf{A}_{\mathbf{1}}\right\} \\
& \beta_{2}=\operatorname{tr}\left\{\tilde{\mathbf{M}}_{\mathbf{2}} \mathbf{M}_{\mathbf{0}}{ }^{-1}\right\}=\operatorname{tr}\left\{\mathbf{B}_{\mathbf{1}}\right\} \\
& \beta_{3}=\operatorname{tr}\left\{\tilde{\mathbf{M}}_{\mathbf{3}} \mathbf{M}_{\mathbf{0}}{ }^{-1}\right\}=\operatorname{tr}\left\{\mathbf{A}_{\mathbf{2}}\right\} \\
& \beta_{4}=\operatorname{tr}\left\{\tilde{\mathbf{M}}_{\mathbf{4}} \mathbf{M}_{\mathbf{0}}{ }^{-1}\right\}=\operatorname{tr}\left\{\mathbf{B}_{\mathbf{2}}\right\} \\
& \beta_{5}=\operatorname{tr}\left\{\tilde{\mathbf{M}}_{\mathbf{2}} \mathbf{M}_{\mathbf{0}}{ }^{-1} \tilde{\mathbf{M}}_{\mathbf{1}} \mathbf{M}_{\mathbf{0}}{ }^{-1}\right\}=\operatorname{tr}\left\{\mathbf{A}_{\mathbf{1}} \mathbf{B}_{\mathbf{1}}\right\} \\
& \beta_{6}=\operatorname{tr}\left\{\tilde{\mathbf{M}}_{\mathbf{4}} \mathbf{M}_{\mathbf{0}}{ }^{-1} \tilde{\mathbf{M}}_{\mathbf{3}} \mathbf{M}_{\mathbf{0}}{ }^{-1}\right\}=\operatorname{tr}\left\{\mathbf{A}_{\mathbf{2}} \mathbf{B}_{\mathbf{2}}\right\}
\end{aligned}
$$

$$
\begin{aligned}
& \beta_{7}=\operatorname{tr}\left\{\tilde{\mathbf{M}}_{3} \mathbf{M}_{\mathbf{0}}{ }^{-1} \tilde{\mathbf{M}}_{\mathbf{1}} \mathbf{M}_{\mathbf{0}}{ }^{-1}\right\}=\operatorname{tr}\left\{\mathbf{A}_{\mathbf{2}} \mathbf{L} \mathbf{A}_{\mathbf{1}} \mathbf{L}^{-1}\right\} \\
& \beta_{8}=\operatorname{tr}\left\{\tilde{\mathbf{M}}_{\mathbf{3}} \mathbf{M}_{\mathbf{0}}{ }^{-1} \tilde{\mathbf{M}}_{\mathbf{2}} \mathbf{M}_{\mathbf{0}}{ }^{-1}\right\}=\operatorname{tr}\left\{\mathbf{A}_{\mathbf{2}} \mathbf{L} \mathbf{B}_{\mathbf{1}} \mathbf{L}^{-1}\right\} \\
& \beta_{9}=\operatorname{tr}\left\{\tilde{\mathbf{M}}_{\mathbf{4}} \mathbf{M}_{\mathbf{0}}{ }^{-1} \tilde{\mathbf{M}}_{\mathbf{1}} \mathbf{M}_{\mathbf{0}}{ }^{-1}\right\}=\operatorname{tr}\left\{\mathbf{B}_{\mathbf{2}} \mathbf{L} \mathbf{A}_{\mathbf{1}} \mathbf{L}^{-1}\right\} \\
& \beta_{10}=\operatorname{tr}\left\{\tilde{\mathbf{M}}_{\mathbf{4}} \mathbf{M}_{\mathbf{0}}{ }^{-1} \tilde{\mathbf{M}}_{\mathbf{2}} \mathbf{M}_{\mathbf{0}}{ }^{-1}\right\}=\operatorname{tr}\left\{\mathbf{B}_{\mathbf{2}} \mathbf{L} \mathbf{B}_{\mathbf{1}} \mathbf{L}^{-1}\right\}
\end{aligned}
$$

With Eqs. (47) and (48) the following relations for the reflection coefficient $\rho_{a}$ and $\rho_{b}$ result:

$\rho_{a}^{2}=-\beta_{1} \mu_{f a 1}+\Delta m_{a 1}^{2} \mu_{f a 1}^{2}+1$
$\rho_{b}^{2}=-\beta_{2} \mu_{f b 1}+\Delta m_{b 1}^{2} \mu_{f b 1}^{2}+1$

From Eqs. (47) to (50) it can be derived:

$\mu_{f a 2}=\frac{\beta_{1}}{\beta_{3}} \mu_{f a 1}, \quad \mu_{f b 2}=\frac{\beta_{2}}{\beta_{4}} \mu_{f b 1}$

For the obstacle parameter $\mu_{f b 1}$ it can be found:

$$
\begin{aligned}
\mu_{f b 1} & =\frac{m_{5} \mu_{f a 1}\left(1-0.5 \beta_{1} \mu_{f a 1}\right)}{m_{1} \mu_{f a 1}{ }^{2}+m_{2} \mu_{f a 1}+m_{3}} \\
m_{1} & =\Delta m_{a 1}^{2} \beta_{1}\left(\beta_{8} / \beta_{3}-\beta_{2}\right)-0.5 \beta_{1}\left(\beta_{7} / \beta_{3}-\beta_{1}\right)\left(\beta_{1} \beta_{2}-\beta_{5}\right) \\
m_{2} & =-\beta_{1} m_{3}+0.5 \beta_{2} m_{5} \\
m_{3} & =\beta_{8} \beta_{1} / \beta_{3}-\beta_{5} \\
m_{5} & =\beta_{7} \beta_{1} / \beta_{3}-\beta_{1}^{2}+2 \Delta m_{a 1}^{2}
\end{aligned}
$$

and for the line parameter $k$ the equation results:

$$
\left(k-k^{-1}\right)^{2}=\frac{m_{5} \mu_{f a 1}^{2}}{-1+\beta_{1} \mu_{f a 1}-\Delta m_{a 1}^{2} \mu_{f a 1}^{2}}
$$

After some algebraic manipulation a quadratic equation for the obstacle parameter $\mu_{f a 1}$ can be derived:

$$
\begin{aligned}
& m_{8} \mu_{f a 1}^{2}+m_{9} \mu_{f a 1}+m_{10}=0 \\
m_{6}= & \beta_{10} \beta_{2} / \beta_{4}-\beta_{2}^{2}+2 \Delta m_{b 1}^{2} \\
m_{7}= & m_{5} \Delta m_{b 1}-m_{6} \Delta m_{a 1} \\
m_{8}= & 0.25 \beta_{1}^{2} m_{5} m_{7}+0.5 \beta_{1} \beta_{2} m_{1} m_{5}+m_{1}^{2} \\
m_{9}= & m_{5}\left(\beta_{1}\left(0.25 \beta_{1}^{2} m_{6}-m_{7}+0.5 \beta_{2} m_{2}\right)-\beta_{2} m_{1}\right) \\
& +2 m_{1} m_{2} \\
m_{10}= & m_{5}\left(\beta_{1}\left(-1.25 \beta_{1} m_{6}+0.5 \beta_{2} m_{3}\right)+m_{7}-\beta_{2} m_{2}\right) \\
& +m_{2}^{2}+2 m_{1} m_{3}
\end{aligned}
$$

The unknown parameters of the $\mathrm{LR}_{1} \mathrm{R}_{2}$-method can thus be calculated within the self-calibration procedure. In order to choose the correct solution, an approximate knowledge of the geometrical dimensions is necessary. Besides this solution on the basis of five calibration structures a further solution based on only four structures is also possible.

\section{TRM-method}

Based on the setup in Fig. 6, the theory of the reflective calibration methods without transmission can be transfered to the well-known TRM-method. The calibration standards of the TRM method consist of a reflection with the reflection 

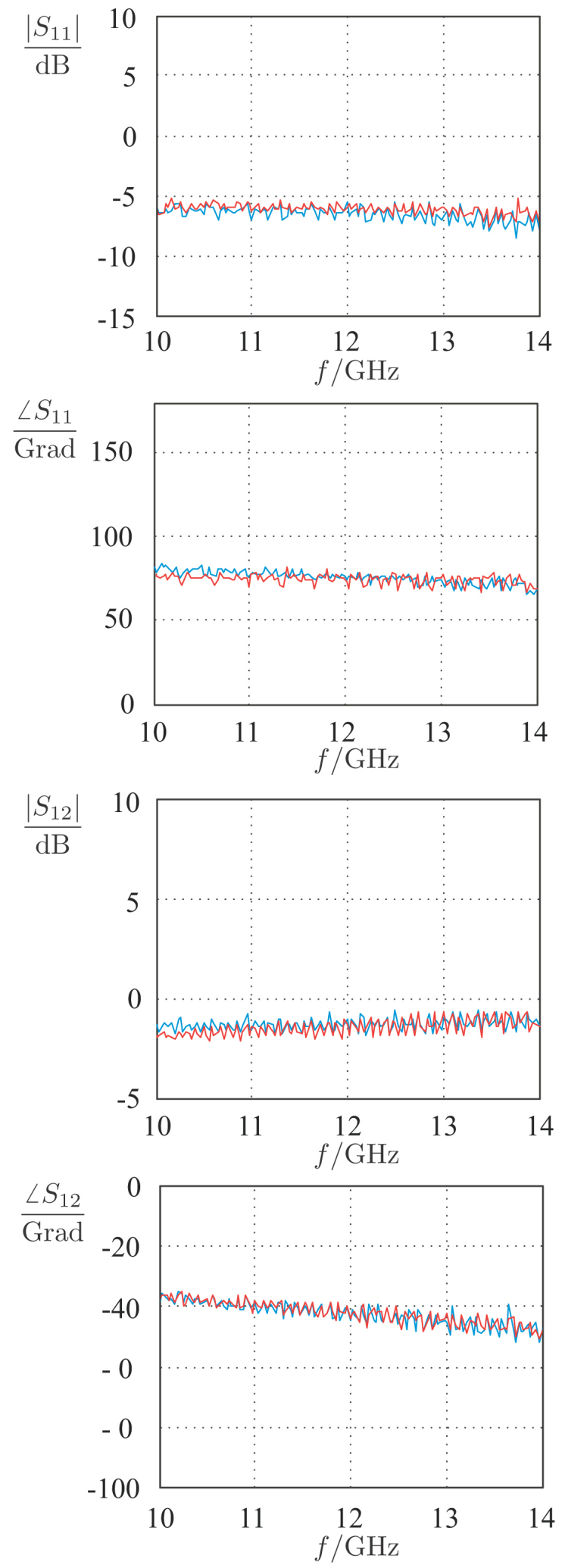

Fig. 8. Error-corrected scattering parameters of the material according to the TRL (blue line) and the LRR method (red line).
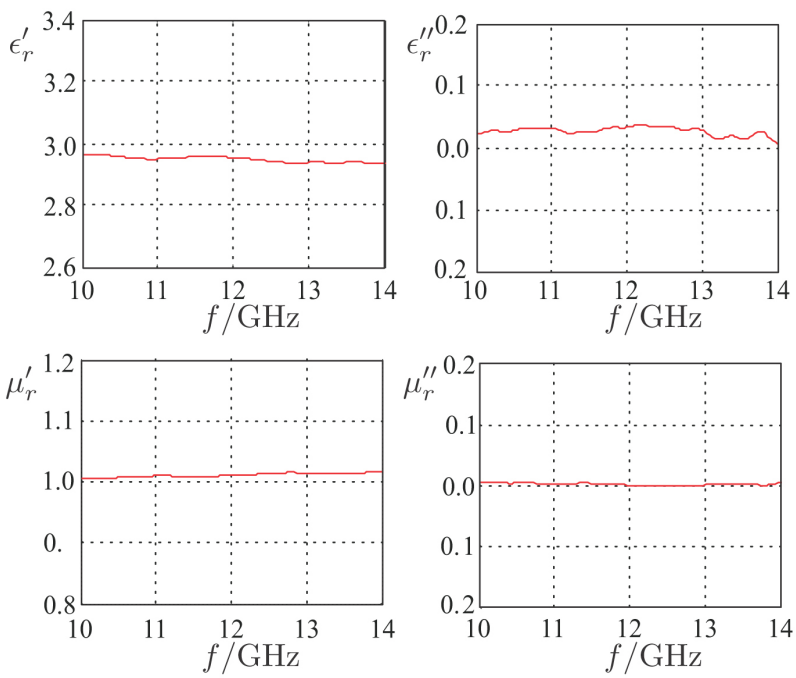

Fig. 9. Measured permittivity and permeability.

coefficient $\rho$ and of a match with the reflection coefficient $\delta$ which is supposed to converge towards zero. One possible realization of the match in a free space system, based on a mirror and an absorber, is depicted in Fig. 7.

With the bilinear relations of Eqs. (21) and (25) where the measurement matrix $\mathbf{M}_{\mathbf{0}}$ corresponds to the T-standard and $k$ equals one, because the T-standard is supposed to be of length zero, the following cross relation can be written with $\rho_{l, 1}=\rho_{r, 1}=\rho$ for the R-standard and $\rho_{l, 2}=\rho_{r, 2}=\delta$ for the M-standard:

$$
\begin{aligned}
v & =\frac{\left(v_{l, 2}-v_{l, 1}\right)\left(v_{r, 2}-v_{r, 1}\right)}{\left(v_{l, 2}-v_{r, 1}\right)\left(v_{r, 2}-v_{l, 1}\right)}=\frac{(\delta-\rho)\left(\delta^{-1}-\rho^{-1}\right)}{\left(\delta-\rho^{-1}\right)\left(\delta^{-1}-\rho\right)} \\
& =\frac{(\delta-\rho)\left(1-\delta \rho^{-1}\right)}{\left(\delta-\rho^{-1}\right)(1-\delta \rho)}
\end{aligned}
$$

With $\delta=0$ for the match the very compact solution for the reflection coefficient $\rho$ results:

$v=\rho^{2}$

\section{Experimental results}

Measurements were performed with a free space system in a frequency range from $10 \mathrm{GHz}$ to $14 \mathrm{GHz}$. Some measurement results for a polyamide probe (P6, thickness: $4.2 \mathrm{~mm}$, size: $50 \mathrm{~cm} \times 50 \mathrm{~cm}$ ) are shown in the following.

In Fig. 8 the error-corrected scattering parameters calculated according to the TRL in comparison to the LRR method are depicted. Both methods show a good agreement. Based on the measured scattering parameters the calculated permittivity is depicted in Fig. 9. 


\section{Conclusion}

Different methods for the calibration of vector network analyzers are presented. The calibration structures are principally based on obstacle networks with either transmission, no transmission or only a weak transmission. All methods have in common that the calibration structures are all of equal mechanical length. They are thus well suited for the implementation in a free space system for the determination of materials dielectric properties at microwave frequencies. The robust functionality of the methods is confirmed in measurements.

\section{References}

Ghodgaonkar, D. K., Varadan, V. V., and Varadan, V. K.: A FreeSpace Method for Measurement of Dielectric Constants and Loss Tangents at Microwave Frequencies, IEEE Trans. Instrum. Meas., 37, 789-793, June, 1989.

Engen, G. F. and Hoer, C. A.: Thru-Reflect-Line: An improved technique for calibrating the dual six port automatic network analyzer, IEEE Trans. Microw. Theory Tech., 27, 987-993, Dec., 1979.

Eul, H.-J. and Schiek, B.: A Generalized Theory and New Calibration Procedures for Network Analyzer Self-Calibration, IEEE Trans. Microw. Theory Tech., 39, 724-731, April, 1991.

Heuermann, H. and Schiek, B.: Line Network Network (LNN): An Alternative In-Fixture Calibration Procedure, IEEE Trans. Microw. Theory Tech., 45, 408-413, March, 1997.

Rolfes, I. and Schiek, B.: Calibraton Methods for Free Space Dielectric Microwave Measurements with a 4-Channel-NetworkAnalyzer, in Proc. 32nd EUMC, 1077-1080, 2002.

Rolfes, I. and Schiek, B.: LRR - A Self-Calibration Technique for the Calibration of Vector Network Analyzers, IEEE Trans. Instrum. Meas., 52, 316-319, April, 2003. 